\title{
Hyperinsulinemia
}

\section{A Link Between Hypertension Obesity and Glucose Intolerance}

\author{
Michaela Modan, Hillel Halkin, Shlomo Almog, Ayala Lusky, Aliza Eshkol, Menachem Shefi, \\ Angela Shitrit, and Zahava Fuchs \\ Departments of Clinical Epidemiology and Medicine, Clinical Pharmacology Unit, and Institute of Endocrinology, \\ Chaim Sheba Medical Center, Tel Hashomer, affiliated with the Tel Aviv University Sackler Medical School, Israel
}

\begin{abstract}
Hypertension and glucose intolerance, determined in a random population sample $(n=2,475)$, showed a highly significant $(P$ $<0.001$ ) association from the mildest levels of both conditions, independent of the confounding effects of age, sex, obesity, and antihypertensive medications. Summary rate ratios for hypertension were 1.48 (1.18-1.87) in abnormal tolerance and 2.26 (1.69-2.84) in diabetes compared with normal tolerance. Altogether, $83.4 \%$ of the hypertensives were either glucoseintolerant or obese-both established insulin-resistant conditions. Fasting and post-load insulin levels in a representative subgroup $(n=1,241)$ were significantly elevated in hypertension independent of obesity, glucose intolerance, age, and antihypertensive medications. The mean increment in summed 1- and 2-h insulin levels (milliunits per liter) compared with nonobese normotensives with normal tolerance was 12 for hypertension alone, 47 for obesity alone, 52 for abnormal tolerance alone, and 124 when all three conditions were present. The prevalence of concentrations (milliequivalents per liter) of erythrocyte $\mathrm{Na}^{+} \geq 7.0, \mathrm{~K}^{+}<92.5$, and plasma $\mathrm{K}^{+} \geq 4.5$ in a subsample of 59 individuals with all combinations of abnormal tolerance obesity and hypertension was compared with those in 30 individuals free of these conditions. Altogether, $88.1 \%$ of the former vs. $40.0 \%$ of the latter group presented at least one of these three markers of internal cation imbalance $(P<0.001)$. We conclude that insulin resistance and/or hyperinsulinemia (a) are present in the majority of hypertensives, (b) constitute a common pathophysiologic feature of obesity, glucose intolerance, and hypertension, possibly explaining their ubiquitous association, and (c) may be linked to the increased peripheral vascular resistance of hypertension, which is putatively related to elevated intracellular sodium concentration.
\end{abstract}

\section{Introduction}

Hypertension, obesity, and glucose intolerance (impaired glucose tolerance and noninsulin-dependent diabetes) are so commonly associated (1-9) as to suggest common pathogenetic mechanisms. Impaired glucose tolerance and obesity are characterized by hyperinsulinemia, which reflects peripheral insulin

Address correspondence to Dr. Modan, Head, Biometry Unit, Department of Clinical Epidemiology, Chaim Sheba Medical Center, Tel Hashomer 52621, Israel.

Received for publication 3 November 1983 and in revised form 16 October 1984.

J. Clin. Invest.

(C) The American Society for Clinical Investigation, Inc.

0021-9738/85/03/809/09 \$1.00

Volume 75, March 1985, 809-817 resistance. Noninsulin-dependent diabetes is also an insulinresistant state, although insulin response may be in the normal range or lower (10-14). Although there are not many direct studies of insulin response in hypertension (2), hyperinsulinemia has been ascribed a pathogenetic role in obese hypertension via increased renal sodium retention (15). Abnormalities of cell membrane cation transport were demonstrated in obesity and hypertension (elevated intracellular sodium) (16-21) and in insulin-dependent diabetes (reduced intracellular and elevated plasma potassium concentrations) $(22,23)$. These, as well as the obligatory linkage of cellular sodium efflux and potassium influx (24) and the regulatory role of insulin in cell membrane cation transport $(22,25)$, raise the possibility that such shifts in internal cation distribution are associated with insulin resistance and/or hyperinsulinemia (23).

These data suggest that nonobese hypertension might also be an insulin-resistant state, and insulin resistance and/or hyperinsulinemia might be a common pathophysiological mechanism linking glucose intolerance, obesity, and hypertension, the latter through changes in internal cation distribution. To test this hypothesis we studied a large representative sample of the adult Jewish population in Israel, a population with high prevalence of diabetes and glucose intolerance $(4-6,26)$ to determine $a$ ) the prevalence of hypertension, from its mildest level, in the entire spectrum of glucose tolerance, and of glucose intolerance from its mildest degree, in the full range of blood pressure, allowing for the effects of obesity, age, and antihypertensive medications, which are the major confounders of this association $(4,6,27-30), b)$ the independent association of hypertension with insulin response, singly and combined with obesity and glucose intolerance, and $c$ ) plasma and erythrocyte sodium and potassium concentrations and their correlation with insulin response in all combinations of obesity, hypertension, and glucose intolerance.

\section{Methods}

Participants and clinical procedures. The current report addresses a subgroup of the Israel Study of Glucose Intolerance, Obesity, and Hypertension. This is an ongoing nationwide longitudinal study of a sample of 5,711 individuals whose names were drawn from the Israel Central Population registry, born between 1912 and 1941 (26).

Between 1977 and 1982, 2,769 participants, a representative group of the original sample, attended regional medical centers after having blood pressure and regular use of medications (verified by inspection of drug receptacles) recorded at home. Blood pressure (millimeters of mercury) was measured with a standard mercury sphygmomanometer in the sitting position. Four blood pressure measurements were obtained, two before and two after the interview. Weight, height, and fasting plasma glucose were determined at the medical center. If participants were not known to be diabetic, they were asked to undergo an oral glucose tolerance test. From 1979 to 1982, determination of insulin response to the oral glucose load was added to the protocol (26). 


\section{Laboratory procedures}

Plasma glucose and serum creatinine levels (milligrams per deciliter) were measured by routine automated Autoanalyzer II (Technicon Instruments Corp., Tarrytown, NY), glucose based on potassium ferricyanide reduction and creatinine on the picric acid method. Plasma insulin (milliunits per liter) was determined in duplicate by Phadebas Radioimmunoassay (Pharmacia Inc., Piscataway, NJ), the within-assay coefficient of variation being $4 \%$, and the between-assay coefficient, $8 \%$. Plasma and erythrocyte cation concentrations as markers of membrane cation transport activity (31) were determined by atomic absorption spectrophotometer (18). Within-assay coefficient of variation was $<0.7 \%$ for all cation determinations. Between-assay coefficients of variation for potassium at 5 and $100 \mathrm{meq} /$ liter were 1.7 and $1.3 \%$, respectively, and for sodium at 7 and $140 \mathrm{meq} / \mathrm{liter}$, were 3.3 and $1.9 \%$. The laboratory staff was unaware of the clinical condition of the examinees at the time of determination.

\section{Definitions}

Age. Age groups were defined by decade of year of birth, since the original sample was obtained by sampling separately from each of the three population strata; 1932-1941, 1922-1931, and 1912-1921. Participant ages at the time of examination ranged between 35 and 70 years.

Obesity. Categories included nonobese, body mass index (weight/ height ${ }^{2}, \mathrm{~kg} / \mathrm{m}^{2}$ ) $<25$ (satisfactory weight or mild overweight); and obese, body mass index $\geq 25$ (moderate to severe overweight) (29).

Blood pressure. Categories included untreated hypertension, at least two of the four measurements with either systolic $>145$ or diastolic >93; treated hypertension, reported use of antihypertensive drugs, including diuretics; and normotensive, all remaining individuals.

Glucose tolerance. Glucose tolerance was classified according to the National Diabetes Data Group criteria (29) into normal, borderline (intermediate combinations between normal and impaired tolerance; the nondiagnostic group by these criteria), and impaired, as well as diabetic. The diabetes group included newly found cases by the glucose tolerance test and previously known, treated diabetics. We defined glucose intolerance as the whole group with non-normal tolerance, and abnormal tolerance as the group including only borderline and impaired glucose tolerance. A $100-\mathrm{g}$ oral glucose load was used instead of the recommended $75 \mathrm{~g}$ for its greater stimulation of insulin response (32). The difference between loads had little effect on blood glucose levels (29).

Hyperinsulinemia. Hyperinsulinemia was defined as fasting or sum of insulin levels 60 and $120 \mathrm{~min}$ post load (sum insulin) above the 75th percentile of the sum insulin distribution in the reference group, namely nonobese normotensives with normal tolerance $\left(\mathrm{PN}_{75}\right)$.

\section{Sampling of subjects for cation measurements}

The nondiabetics among individuals in whom insulin was determined were classified into eight categories; a reference group and seven diagnostic strata representing all possible combinations of obesity, hypertension, and abnormal tolerance. Lists of consecutive patients belonging to each of the eight strata were compiled with the aim of recruiting 30 individuals of the reference group, and 70 individuals equally distributed among the other seven strata.

\section{Data analysis}

Association of hypertension and glucose intolerance. Univariate testing of the increasing rate of all categories of glucose intolerance in the three blood pressure categories was done by the $\chi^{2}$ test for linear trends. Multivariate analysis allowing for confounding variables of the association of glucose intolerance and blood pressure level was performed in two ways: (a) analysis of covariance (Biomedical Computer Programs, University of California, 1981 program 2V): dependent variablessystolic and diastolic blood pressure, respectively; grouping factorssex and glucose tolerance (in three categories-normal, abnormal, and diabetes, both untreated and treated); continuous covariates-accounting for the linear effect of age and body mass index, and (b) Mantel-
Haenszel statistic with test based 95\% confidence limits (33) comparing the ratio of the rate of hypertensives in the abnormal tolerance group with that in the normal tolerance group, and the same ratio in the diabetics to that in the normal tolerance group. Both analyses were done in strata by age and body mass index, testing for homogeneity of risks across all strata.

Effect of hypertension on insulin response. This effect was analyzed in the insulin subset in two ways: $(a)$ analysis of covariance: dependent variables-log transformed values of fasting insulin level and of sum insulin, respectively; grouping factors-blood pressure level (in three categories) and glucose tolerance (in three categories as above, but including only newly found diabetics, as previously known diabetics did not undergo glucose tolerance testing); covariates-age and body mass index; and $(b)$ Mantel-Haenszel statistic analyzing the ratio of the rates of persons with fasting or sum insulin $\geq \mathrm{PN}_{75}$ among hypertensives and normotensives in strata by glucose tolerance level and body mass index as above.

All analyses in the total study group and the insulin subset were performed twice; first on all persons, and again excluding persons receiving antihypertensive drugs. The analyses of covariance included all first-order interaction terms, testing the homogeneity of the association of the dependent variable with each of the grouping factors across all strata defined by the other grouping factors. Test based $95 \%$ confidence limits for summary rate ratios (33) appear in brackets following the rate ratios throughout the text.

Analysis of internal cation distribution. The distributions of plasma and erythrocyte sodium and potassium concentrations were dichotomized by cutoff points selected to maximize the discrimination between the reference group and diagnostic strata. Differences in these distributions between individuals composing the reference group and those in the diagnostic strata were analyzed by Fisher's exact test for $2 \times 2$ tables.

\section{Results}

General. In 294 of the 2,769 participants, glucose tolerance remained undetermined due to refusals or technical problems. The remaining 2,475 persons (total study group) included 191 previously known diabetics and 2,284 who underwent a full oral glucose tolerance test. Among the latter, insulin response was determined in 1,241 cases.

Of the total study group, $966(39.0 \%)$ were glucose intolerant, including $616(24.9 \%)$ with abnormal glucose tolerance and $350(14.1 \%)$ with overt diabetes, 159 of whom were unaware of this condition before the study. Of the previously known diabetics, 135 were receiving medication, namely oral hypoglycemic drugs $(86 \%)$ or insulin $(14 \%)$, and the rest were treated by diet alone. Altogether, $97 \%$ of the 191 previously known diabetics were noninsulin dependent. Cases classified as hypertensive numbered $1,105(44.6 \%)$. Of these, 711 were untreated while 394 reported regular use of antihypertensive medications as follows: thiazide diuretics, $31 \%$; betadrenergic blocking agents, $33 \%$; combinations of the two, $16 \%$; and other drugs, $20 \%$. Obesity was found in $58.5 \%$. There was a strong association between hypertension, glucose intolerance, and obesity with considerable overlap between the three populations. Of the 966 glucose intolerant, $60.6 \%$ were hypertensive, while $52.9 \%$ of the hypertensives were glucose intolerant. Obesity was present in $69.0 \%$ of the hypertensives compared with $50.0 \%$ of the normotensives, and altogether $83.4 \%$ of the hypertensives were either glucose intolerant or obese.

Association of glucose intolerance and hypertension. Glucose intolerance and hypertension were strongly associated from their mildest levels in the total study group (Table I). The total rate of all categories of glucose intolerance increased 
Table I. Distribution of Glucose Tolerance by Blood Pressure Categories in the Total Study Group

\begin{tabular}{|c|c|c|c|c|c|c|c|}
\hline & \multirow{2}{*}{$\frac{\text { Total }}{\text { No. }}$} & \multicolumn{2}{|c|}{ Normotensives } & \multicolumn{2}{|c|}{ Untreated hypertensives } & \multicolumn{2}{|c|}{ Treated hypertensives } \\
\hline & & No. & $\%$ & No. & $\%$ & No. & $\%$ \\
\hline Total & 2,475 & 1,370 & 100.0 & 711 & 100.0 & 394 & 100.0 \\
\hline Normal tolerance & 1,509 & 989 & 72.2 & 369 & 51.9 & 151 & 38.3 \\
\hline Glucose intolerance: total & 966 & 381 & 27.8 & 342 & 48.1 & 243 & 61.7 \\
\hline Abnormal tolerance: total & 616 & 281 & 20.6 & 198 & 27.9 & 137 & 34.7 \\
\hline Borderline tolerance & 445 & 209 & 15.3 & 137 & 19.3 & 99 & 25.1 \\
\hline Impaired tolerance & 171 & 72 & 5.3 & 61 & 8.6 & 38 & 9.6 \\
\hline Diabetes: total & 350 & 100 & 7.2 & 144 & 20.2 & 106 & 27.0 \\
\hline Newly found & 159 & 47 & 3.4 & 70 & 9.8 & 42 & 10.7 \\
\hline Previously known & 191 & 53 & 3.8 & 74 & 10.4 & 64 & 16.3 \\
\hline \multicolumn{8}{|c|}{ Percent diabetics among the glucose } \\
\hline intolerant & & \multicolumn{2}{|c|}{26.2} & \multicolumn{2}{|c|}{42.1} & \multicolumn{2}{|c|}{43.6} \\
\hline \multicolumn{8}{|l|}{ Mean \pm SD blood pressure* } \\
\hline Systolic & & \multicolumn{2}{|c|}{$119.1 \pm 11.9$} & \multicolumn{2}{|c|}{$147.7 \pm 18.3$} & \multicolumn{2}{|c|}{$152.6 \pm 23.3$} \\
\hline Diastolic & & \multicolumn{2}{|c|}{$78.1 \pm 7.2$} & \multicolumn{2}{|c|}{$91.5 \pm 10.1$} & \multicolumn{2}{|c|}{$93.3 \pm 13.0$} \\
\hline
\end{tabular}

* The large SD in the hypertensives reflects the distribution's skew toward high values. In addition, some cases were defined as hypertensive on the basis of elevated systolic with normal diastolic pressure and vice versa.

significantly from $27.8 \%$ in the normotensives to $48.1 \%$ in untreated hypertensives and to $61.7 \%$ in the treated hypertensives. This overall trend, evident in each of the intolerance categories, was highly significant $(P<0.0001)$. Moreover, glucose intolerance was not only more frequent but also more severe among hypertensives. This is expressed by the marked increase $(P<0.0001)$ in the proportion of diabetics among the glucose-intolerant, which rose from $26.2 \%$ in the normotensives to 42.1 and $43.6 \%$ in the untreated and treated hypertensives, respectively. The difference in severity of glucose intolerance between treated and untreated hypertensives was not significant. Note that the association between glucose intolerance and hypertension was evident even at the mildest levels of both. Hypertension was mild among untreated hypertensives (147.7/91.5 mean blood pressure), while the ratio of the rates of abnormal tolerance in the untreated hypertensives and in normotensives was 1.4 , a highly significant difference $(P<0.001)$.

The association of glucose intolerance and hypertension was independent of the effect of age, obesity, and use of antihypertensive medications. After excluding the 394 treated hypertensives and stratifying the remaining untreated subset by age and body mass index (Fig. 1), the means of the systolic and diastolic blood pressure levels and the rate of hypertensives were compared in three categories of glucose tolerance; normal, abnormal, and diabetes, both newly found and previously known. In addition to the expected increase with increasing age $(P<0.0001)$ and body mass index $(P<0.0001)$, both systolic and diastolic pressures increased consistently with increasing glucose intolerance in all but one of the strata. This effect of glucose intolerance was highly significant $(P<0.0001)$ for both pressures, and was present in both sexes. When only the normal and abnormal tolerance groups were compared, diabetics excluded, differences were still significant for systolic $(P<0.0001)$ and diastolic $(P<0.001)$ pressures. In comparing the diabetic and abnormal tolerance groups, there was a significant difference in systolic $(P<0.0001)$ but not in diastolic pressure $(P=0.15)$. Findings were similar with respect to the rate of categorically defined hypertension, which increased appreciably from the normal to the abnormal tolerance and from the latter to the diabetic group, in all age and body mass index strata.

The degree of increase in risk of hypertension with increasing glucose intolerance was expressed in all age and body mass index strata by the ratios of the rates of hypertension in the abnormal tolerance and in the diabetics to those in the normal

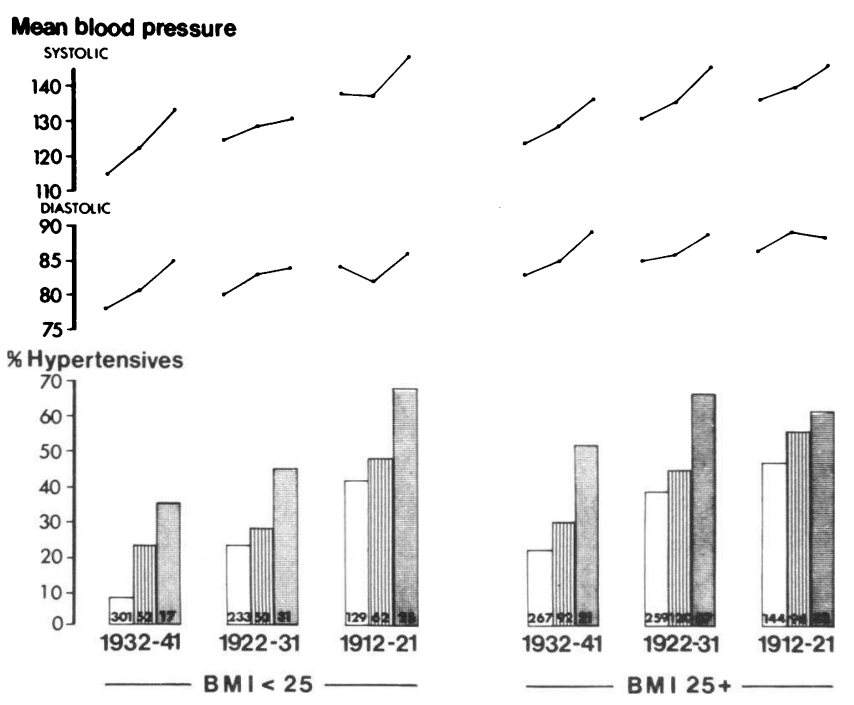

Figure 1. Mean systolic and diastolic blood pressure and rate of hypertensives by body mass index (BMI), age by birth year, and glucose tolerance in the untreated subset (normotensives and untreated hypertensives). Open bars, normal tolerance; hatched bars, abnormal tolerance; stippled bars, diabetes (both newly found and previously known). The figure does not include 42 individuals of the untreated subset whose body mass index was not recorded. 
Table II. Ratio of Rate of Hypertensives in Abnormal Tolerance and in Diabetes to That in Normal Tolerance, by Age and Body Mass Index in the Total Study Group and Untreated Subset (Normotensives and Untreated Hypertensives)

\begin{tabular}{|c|c|c|c|c|c|}
\hline \multirow[b]{2}{*}{ Year of birth } & \multirow[b]{2}{*}{ Group } & \multicolumn{2}{|l|}{$\mathrm{BMI}^{*}<25.0$} & \multicolumn{2}{|l|}{ BMI $25.0+$} \\
\hline & & Abnormal tolerance & Diabeles & Abnormal tolerance & Diabetes \\
\hline \multirow[t]{2}{*}{$1932-1941$} & Total & 2.29 & 3.23 & 1.28 & 2.03 \\
\hline & Untreated & 2.89 & 4.43 & 1.40 & 2.41 \\
\hline \multirow[t]{2}{*}{$1922-1931$} & Total & 1.41 & 2.02 & 1.23 & 1.59 \\
\hline & Untreated & 1.21 & 1.95 & 1.14 & 1.69 \\
\hline \multirow[t]{2}{*}{$1912-1921$} & Total & 1.24 & 1.65 & 1.28 & 1.37 \\
\hline & Untreated & 1.16 & 1.62 & 1.21 & 1.35 \\
\hline
\end{tabular}

* BMI, body mass index.

tolerance group. All ratios were considerably $>1$ (Table II). The effect of glucose intolerance was strongest in the nonoverweight youngest age group in which the rate of hypertension rose more than twofold in individuals with abnormal tolerance and more than threefold in diabetics. In the other age and body mass index groups, the rate of hypertension was increased by $20-40 \%$ in abnormal tolerance and by $40-100 \%$ in the diabetics. The differences in risks between the age-body mass index strata, however, were not significant for impaired tolerance $(P>0.20)$ and diabetes $(P>0.30)$. Treated hypertensives excluded, the summary rate ratio of hypertension in abnormal as compared with normal tolerance was 1.48 (1.18-1.87), and in diabetics as compared with the normal group was 2.26 (1.69-2.84). The diabetics also differed significantly from the abnormal tolerance group $(P<0.001)$ with a summary rate ratio of 1.93 (1.39-2.69). The pattern in the total study group was practically the same as in the untreated subset.

Hypertension and plasma insulin. Hypertension was found to be independently associated with hyperinsulinemia (Fig. 2). In all but one of the glucose tolerance and body mass index strata, mean sum insulin and the percentage of individuals with sum insulin $\geq \mathrm{PN}_{75}$ were greater among the hypertensives. The only exceptions were the hypertensive overweight diabetics, whose values were lower than the respective normotensive group. As expected, obesity and abnormal tolerance were also associated with increased insulin response. Moreover, the effects of hypertension, abnormal tolerance, and obesity on sum insulin seemed to be linearly additive. Thus, the mean increment in sum insulin above the reference group was 12 $\mathrm{mU} /$ liter for hypertension alone, $47 \mathrm{mU} /$ liter for obesity alone, $52 \mathrm{mU} /$ liter for abnormal tolerance alone, and $124 \mathrm{mU} / \mathrm{liter}$ for the combination of all three. This linear additivity was present in all combinations of two conditions as well. Analysis of covariance of the total study group showed a significant independent effect of hypertension on sum insulin $(P<0.01)$ when the effect of body mass index, age, sex, and glucose tolerance were taken into account. Glucose tolerance and body mass index each also had a highly significant effect on sum insulin $(P<0.001)$. Age and sex had no effect $(P>0.20)$. The interaction term between glucose tolerance and blood pressure level was significant $(P=0.03)$, reflecting the reversal of the trend in the overweight diabetics. Similar results were obtained for fasting insulin. A significant independent increase was associated with hypertension $(P=0.04)$, with a significant interaction $(P=0.02)$ between glucose tolerance and blood pressure level, the expected highly significant increase due to overweight and glucose intolerance $(P<0.0001)$ and no effect of age or sex $(P>0.30)$. Results for the untreated subset for sum and fasting insulin were essentially the same.

The effect of hypertension was strongest in the normal tolerance group as demonstrated by comparing the ratio of rates of persons with sum insulin $\geq \mathrm{PN}_{75}$ in hypertensives with those in normotensives in all body mass index and glucose tolerance strata (Table III). Thus, the rate of sum insulin $\geq \mathrm{PN}_{75}$ was increased almost 1.5 -fold in the nonoverweight, and 1.2-fold in the overweight with normal tolerance. The effect was smaller in abnormal tolerance and was reversed in

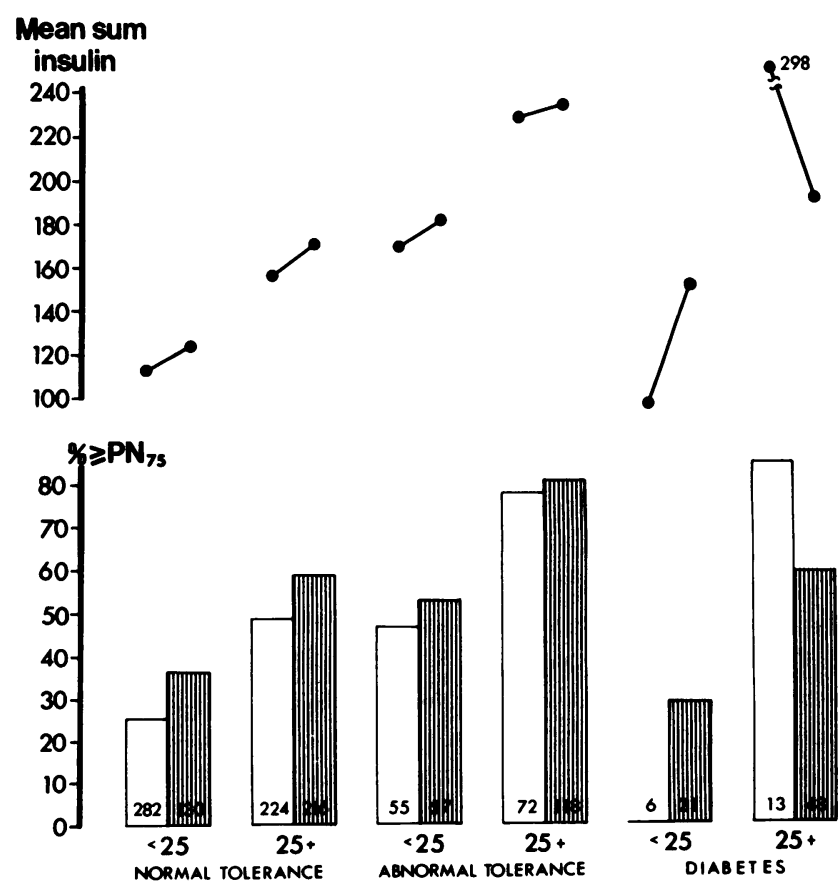

Figure 2. Comparison of rate of individuals with sum insulin $\geq \mathrm{PN}_{75}$ and of mean sum insulin in hypertensives and normotensives by body mass index and glucose tolerance. Open bars, normotensives; hatched bars, hypertensives. Only newly found diabetics are included. (Lack of bar for normotensive diabetics with body mass index (BMI) $<25$ reflects zero rate). 
Table III. Ratio of Rates of Sum Insulin and Fasting Insulin $\geq P N_{75}$ in Hypertensives to That in Normotensives by Body Mass Index and Glucose Tolerance in the Total Group and Untreated Subset (Normotensives and Untreated Hypertensives)

\begin{tabular}{|c|c|c|c|c|c|}
\hline & \multicolumn{2}{|c|}{ Normal tolerance } & \multicolumn{2}{|c|}{ Abnormal tolerance } & \multirow{2}{*}{$\frac{\text { Diabetes }^{*}}{25+}$} \\
\hline & $<25$ & $25+$ & $<25$ & $25+$ & \\
\hline \multicolumn{6}{|c|}{ Sum insulin $\geq \mathbf{P N}_{75}$} \\
\hline Total & 1.45 & 1.21 & 1.11 & 1.04 & 0.71 \\
\hline Untreated & 1.41 & 1.19 & 1.06 & 1.00 & 0.67 \\
\hline \multicolumn{6}{|c|}{ Fasting insulin $\geq \mathrm{PN}_{75}$} \\
\hline Total & 1.00 & 1.38 & 1.46 & 0.90 & 0.91 \\
\hline Untreated & 1.15 & 1.42 & 1.31 & 0.77 & 0.84 \\
\hline
\end{tabular}

* Only newly found diabetics are included here since in known diabetics glucose tolerance was not tested. As there were only six diabetics with body mass index $<25$, rate ratios were not computed.

the overweight untreated diabetics. The overall higher rate of sum insulin $\geq \mathrm{PN}_{75}$ in hypertensives as compared with normotensives was significant $(P=0.03)$. To exclude the effect of the reversal of the trend in the diabetics, analysis was repeated without them. The excess of persons with sum insulin $\geq \mathrm{PN}_{75}$ in hypertensives continued to be significant $(P<0.01)$. This excess was not due to persons on antihypertensive drugs, since trends were practically identical when they were excluded from the analysis (Table III). The effect of hypertension on the rate of fasting insulin $\geq \mathrm{PN}_{75}$ in the various strata was similar to that on sum insulin, although weaker in the non-overweight with normal tolerance. While the overall effect of hypertension on this rate was not significant $(P=0.13)$, upon reanalysis excluding the diabetics, it approached significance $(P=0.06)$. Results of analysis of covariance are summarized in Table IV.

Plasma and erythrocyte sodium and potassium concentrations. Cation concentrations were determined in 89 individuals of the insulin subset, 30 belonging to the reference group and 59 drawn from the diagnostic strata. The distributions of sodium and potassium concentrations in both erythrocyte and plasma were significantly altered in the group of 59 persons of the diagnostic strata (total hyperinsulinemic group), as compared with the reference group (Table V). A higher proportion of erythrocyte sodium concentrations was in the high range $(\geq 7.0, P=0.02)$, while those of potassium were shifted toward the low range $(<92.5, P=0.05)$. In plasma a higher proportion of concentrations was in the high range for both potassium ( $\geq 4.5, P<0.001)$ and sodium $(\geq 140, P=0.04)$. Since erythrocyte concentrations of sodium and potassium and plasma concentrations of potassium are known to be affected by insulin, the three respective high concentration ranges, discriminating between the hyperinsulinemic and reference groups, were termed markers of internal cation imbalance. Altogether, 52 of 59 individuals $(88.1 \%)$ in the hyperinsulinemic group presented at least one marker as compared with 12 of 30 (40\%) in the reference group $(P<0.0001)$. Findings were essentially the same in $\mathbf{4 5}$ persons not receiving medications. In each of the seven diagnostic strata almost all individuals presented at least one marker. The differences from the reference group were significant in virtually all strata. Note that six out of seven individuals with glucose intolerance alone had at least one marker, differing significantly in themselves from the reference group $(P=0.037)$. In all, 31 out of 34 hypertensives $(P<0.001), 29$ out of 34 glucose-intolerant individuals $(P$ $<0.001)$, and 37 out of 42 overweight individuals $(P<0.001)$ presented at least one marker, differing highly significantly

Table IV. Significance Levels: Analysis of Covariance

\begin{tabular}{|c|c|c|c|c|}
\hline & \multicolumn{4}{|c|}{ Dependent variable } \\
\hline & \multicolumn{2}{|l|}{ Sum insulin } & \multicolumn{2}{|c|}{ Fasting insulin } \\
\hline & Total group & Untreated subset & Total group & Untreated subset \\
\hline \multicolumn{5}{|l|}{ Grouping factors } \\
\hline Sex & 0.21 & 0.07 & 0.38 & 0.31 \\
\hline Hypertension & $<0.01$ & 0.02 & 0.04 & 0.11 \\
\hline Glucose intolerance & $<0.0001$ & $<0.0001$ & $<0.01$ & $<0.01$ \\
\hline Hypertension-glucose intolerance interaction* & 0.03 & $<0.01$ & 0.02 & 0.06 \\
\hline \multicolumn{5}{|l|}{ Covariates } \\
\hline Body mass index & $<0.0001$ & $<0.0001$ & $<0.0001$ & $<0.0001$ \\
\hline Age & 0.37 & 0.33 & 0.55 & 0.70 \\
\hline
\end{tabular}

\footnotetext{
* This interaction term tests whether the association of hypertension with insulin response is similar in all glucose tolerance strata. The signifi-
} cant probability reflects the reversal of the trend in obese diabetics, in whom insulin response is lower in hypertensives. 


\begin{tabular}{|c|c|c|c|c|c|c|}
\hline & \multirow[b]{3}{*}{$\begin{array}{l}\text { Total } \\
\text { examined }\end{array}$} & \multicolumn{3}{|l|}{ Markers } & \multirow[b]{3}{*}{$\begin{array}{l}\text { At least one } \\
\text { marker present }\end{array}$} & \multirow[b]{3}{*}{$\begin{array}{l}P \text { vs. } \\
\text { reference group }\end{array}$} \\
\hline & & \multicolumn{2}{|c|}{ Erythrocyte } & \multirow{2}{*}{$\frac{\text { Plasma }}{\mathrm{K}^{+} \geq 4.5}$} & & \\
\hline & & $\mathrm{Na}^{+} \geq 7$ & $\mathrm{~K}^{+}<92.5$ & & & \\
\hline \multicolumn{7}{|l|}{ Reference group } \\
\hline Number & 30 & 5 & 8 & 6 & 12 & \\
\hline Percent & 100.0 & 16.7 & 26.7 & 20.0 & 40.0 & \\
\hline \multicolumn{7}{|l|}{ Total hyperinsulinemic group } \\
\hline Number & 59 & 26 & 28 & 30 & 52 & \\
\hline Percent & 100.0 & 44.1 & 47.5 & 50.8 & 88.1 & $<0.001$ \\
\hline \multicolumn{7}{|c|}{$\begin{array}{l}\text { Hyperinsulinemic group excluding } \\
\text { treated hypertensives }\end{array}$} \\
\hline Number & 45 & 20 & 20 & 25 & 40 & \\
\hline Percent & 100.0 & 44.4 & 44.4 & 55.6 & 88.8 & $<0.001$ \\
\hline Hypertension & 6 & 3 & 4 & 2 & 5 & 0.067 \\
\hline Overweight & 8 & 4 & 2 & 5 & 7 & 0.021 \\
\hline Glucose intolerance & 7 & 4 & 3 & 4 & 6 & 0.037 \\
\hline Hypertension plus overweight & 11 & 5 & 6 & 7 & 11 & 0.001 \\
\hline Hypertension plus intolerance & 4 & 3 & 2 & 2 & 4 & 0.039 \\
\hline Overweight plus intolerance & 10 & 2 & 4 & 5 & 8 & 0.003 \\
\hline All three risk conditions & 13 & 5 & 7 & 5 & 11 & 0.008 \\
\hline
\end{tabular}

from the reference group. These changes in internal cation distribution could not be ascribed to differences in glomerular filtration rate, as serum creatinine $\geq 1.3 \mathrm{mg} / \mathrm{dl}$ was found in $20 \%$ of the reference group and in $14.3 \%$ of the hyperinsulinemic strata group $(P \geq 0.50)$.

\section{Discussion}

Our findings demonstrate a considerable degree of overlap of the hypertensive and glucose intolerant populations, identify an association of hypertension with hyperinsulinemia, suggest that insulin resistance is a common pathophysiologic feature of obesity, glucose intolerance, and hypertension, explaining their ubiquitous association, and indicate that altered membrane cation transport is a marker of insulin resistance.

Recent major community-based studies have reconfirmed older observations on the increased rate of hypertension in overt diabetes. The association has been shown to be independent of, and additive to, the one expected due to obesity and age $(4,5,7-9)$. Only one of the studies inspected this association in untreated hypertensives (7) despite the known deleterious effect of antihypertensive medication on glucose tolerance (29, $30,34)$. While the association of impaired glucose tolerance with hypertension can be indirectly inferred from a number of epidemiological studies (35-37), the full range of this association was described in only two large-scale community studies $(8,9)$. Both showed a significant continuous rise in mean systolic and diastolic blood pressure from normal through abnormal tolerance to diabetes. None of the studies included the complementary analysis, namely, comparison of levels of glucose tolerance in categories of increasing hypertension. Consequently, the continuity of progressive impairment of glucose tolerance with increasing blood pressure from the mildest levels of both conditions, and the degree of overlap of the hypertensive and glucose-intolerant populations were not fully described. In addition, the effect of antihypertensive medications was not accounted for. Our study shows extensive overlap of the hypertensive and glucose-intolerant populations from their mildest levels. These two conditions thus seem to evolve concomitantly, independently of age, obesity, or antihypertensive medications, strongly suggesting a common pathogenetic mechanism. The higher rate of glucose intolerance in our treated hypertensives can therefore be related not only to the deleterious effect of thiazides and other antihypertensive medications, but to the severity of hypertension as well.

Hypertension is also strongly associated with obesity (13 ), and the latter with increased rates of impaired glucose tolerance as well as of diabetes (3-6). Both obesity and impaired tolerance are characterized by hyperinsulinemia. In these hyperinsulinemic states tissue insulin resistance has been demonstrated by clamp and receptor studies, and the level of insulin response to oral glucose load has been shown to be highly correlated to the level of resistance (10-14). Our finding of an independent increase of insulin response in hypertension suggests that hypertension itself is associated with insulin resistance. The fact that this association was particularly evident in hypertensives (both treated and untreated) with normal tolerance, and was of the same order in the obese and nonobese, underscores this inference.

Direct evidence of insulin resistance or hyperinsulinemia in hypertension, independent of the expected association through obesity and glucose intolerance, is sparse (2). We found only one such report, showing significantly increased insulin response to oral glucose load, in 19 non-obese moderate hypertensives with normal glucose tolerance, 10 of whom were receiving antihypertensive treatment, compared with 46 nor- 
motensive controls (38). Indirect evidence is suggested by the significant positive correlation of fasting insulin with blood pressure independent of obesity in one study in adults (39), and by the positive association of 1-h post load insulin levels with blood pressure in two studies in children $(40,41)$. In these three studies, a concomitant increase in blood glucose was demonstrated but the level of glucose tolerance was not referred to.

Our large-scale population-based study directly demonstrates independently increased insulin response to oral glucose load in hypertension and shows that it occurred even in mild untreated hypertensives.

That insulin resistance could be a pathophysiologic factor shared by obesity, glucose intolerance, and hypertension, is also implied by what is known of the role of insulin in the regulation of membrane cation transport. In vitro studies in a variety of animal tissues have demonstrated the stimulating effect of insulin on cell membrane sodium and potassium transport, independent of its effect on glucose transport (22, $25,42)$. Sodium transport mechanisms have been extensively studied in hypertensive and obese human subjects (16-21). Despite some inconsistency in results, most studies have demonstrated transport abnormalities and increased intracellular sodium concentrations in both conditions. The possible role of insulin resistance in these changes was not addressed in any of these studies. On the other hand, a considerable body of evidence indicates the involvement of insulin in internal potassium balance in humans. Insulin affects potassium uptake by muscle and liver, while insulin deficiency is accompanied by a reduction in intracellular potassium and a rise in its extracellular concentration $(23,42-47)$. Cox et al. (23) reasoned that insulin-resistant states would resemble insulin deficiency in terms of internal potassium distribution. Note that the studies on obesity and hypertension concentrated on sodium, while those on diabetes emphasized potassium transport despite the well established obligatory linkage of cellular sodium efflux and potassium influx (24). The obvious inference is that in the three insulin-resistant states, obesity, glucose intolerance, and hypertension, the pattern of internal cation distribution should be similar.

Indeed, our data show significantly increased erythrocyte sodium, reduced erythrocyte potassium, and increased plasma potassium concentrations not only in hypertension and obesity but also in abnormal glucose tolerance. Note that our findings pertaining to cation distributions did not change when patients receiving antihypertensive drugs were excluded from analysis. Moreover, recently published data indicate that such medications tend to shift erythrocyte sodium back toward normal rather than accentuate the changes associated with hypertension (18).

The sodium hypothesis of hypertension attributes increased peripheral vascular resistance to elevated intracellular sodium concentrations. Based on cross-cultural comparisons, this was thought to be mainly due to increased dietary intake of sodium in salt-sensitive individuals $(16,47-49)$. Within-culture studies indicate, however, that dietary factors account for only a minor segment of the hypertensive population $(50,51)$. Linking the sodium hypothesis to hyperinsulinemia, DeFronzo (15) proposed that hypertension of the obese is caused by increased renal sodium retention promoted by the obese hyperinsulinemia. In view of our findings, we propose that DeFronzo's contention may be extended to nonobese hypertension. Moreover, the evidence cited above suggests that tissue insulin resistance may also play a key role by altering internal sodium and potassium distribution in a direction that is presumably associated with increased peripheral vascular resistance (16, 24,52 ). An alternative mechanism that might be compatible with this pattern is the hyperglycemia that invariably accompanies hyperinsulinemia. This in itself may increase peripheral vascular resistance by increasing intracellular osmolality through enhanced passive diffusion of glucose (40). Another possible link is the stimulatory effect of insulin on the sympathetic nervous system, with its attendant catecholamine excess leading to hypertension and changes in internal cation distribution (8, $53,54)$.

That insulin resistance precedes hypertension, rather than being an outcome of hypertension, is supported by the fact that studies showing that interventions known to improve insulin sensitivity, namely weight reduction, physical activity, and specific diets (mainly low fat and high fiber) (55-59), have been shown in another set of studies to lead to blood pressure reduction (60-65).

The significance of our findings is underscored by the fact that they are based on distributions encompassing the whole range of blood pressure and glucose tolerance in a random population sample. While other mechanisms undoubtedly contribute to the multifactorial etiology of hypertension, insulin resistance is present in the great majority of hypertensives. $83.4 \%$ of our hypertensives were overweight and/or glucoseintolerant, and the remaining $16.6 \%$ were significantly hyperinsulinemic compared with controls. Extrapolation of our conclusions to other populations is supported by the Health and Nutrition Examination Survey study (51), in which $94.5 \%$ of the explained variance of blood pressure were accounted for by increasing age and obesity, which are strongly associated with increasing insulin resistance (glucose intolerance was not included in that analysis).

To reconcile the strong association of obesity, hypertension, and glucose intolerance on the one hand and their separate occurrence on the other, one must assume that each of the three conditions is mediated by different facets of the insulinresistant state $(14,66)$. However, since insulin and its receptors constitute a mutually regulating loop, it is believed that regardless of the original abnormality, once initiated, insulin resistance is self-compounding $(13,14)$. Accordingly, the development of any one of the conditions could lead to the others. The reversal of the trend of increasing hyperinsulinemia with increasing insulin resistance in our obese hypertensive diabetics is in line with the putative eventual $\beta$ cell exhaustion after prolonged hyperinsulinemia (10-12), believed to be the reason for deterioration to noninsulin-dependent diabetes.

Finally, a link with atherosclerotic heart disease is clearly suggested. Obesity, hypertension, and noninsulin-dependent diabetes, as well as impaired glucose tolerance, are established risk conditions for this disease $(3,4,67,68)$. Four recent largescale longitudinal studies in France, Australia, and Finland (69-71) have identified increased insulin response as an independent risk factor. Moreover, dietary and life style risk factors implicated in the etiology of atherosclerotic heart disease-high total caloric intake, reduced fiber intake, increased fat consumption, and reduced physical activity (72-78)-are also associated with increased insulin resistance (55-59). We hope 
that future studies will elucidate to what extent the increased risk for atherosclerotic diseases is due to the presumed atherogenic effect of insulin itself, as claimed by some investigators (79), or to other correlates of the insulin resistant state.

\section{Acknowledgments}

This study was supported by grants from the U.S. National Institutes of Arthritis, Diabetes, Digestive and Kidney Diseases, the Israeli German Fund, the Israel-U.S. Binational Fund, and the Sackler School of Medicine Schreiber Fund.

\section{References}

1. Chiang, B. N., L. V. Perlman, and F. H. Epstein. 1960. Overweight and hypertension: a review. Circulation. 39:403-421.

2. Sims, E. A. H. 1982. Mechanisms of hypertension in the overweight. Hypertension (Dallas). 4(Suppl. III):43-49.

3. Bray, G. A. 1979. Obesity. Dis. Mon. 26(1):1-85.

4. West, K. M. 1978. Epidemiology of diabetes and its vascular lesions. Elsevier/North Holland, New York. 191-284, 351-389.

5. Medalie, J. H., C. M. Papier, U. Goldbourt, and J. B. Herman. 1975. Major factors in the development of diabetes mellitus in 10,000 men. Arch. Intern. Med. 135:811-817.

6. Zimmet, P. 1982. Type 2 (non-insulin-dependent) diabetes: an epidemiological overview. Diabetologia. 22:399-411.

7. Barrett-Connor, E., M. H. Criqui, M. R, Klauber, and M, Holdbrook. 1981. Diabetes and hypertension in a community of older adults. Am. J. Epidemiol. 113:276-284.

8. Jarrett, R. J., H. Keen, M. McCartney, J. H. Fuller, P. J. S. Hamilton, D. D. Reid, and G. Rose. 1978. Glucose tolerance and blood pressure in two population samples: their relation to diabetes mellitus and hypertension. Int. J. Epidemiol. 7:15-24.

9. Butler, W. J., L. D. Ostrander, Jr., W. J. Carman, and D. E. Lamphiear. 1982. Diabetes mellitus in Tecumseh, Michigan: prevalence, incidence, and associated conditions. Am. J. Epidemiol. 116:971-980.

10. Reaven, G. M. 1980. Insulin dependent diabetes mellitus: metabolic characteristics. Metab. Clin. Exp. 29:445-454.

11. Olefsky, J. M., O. G. Kolterman, and J. A. Scarlett. 1982. Insulin action and resistance in obesity and non-insulin dependent type II diabetes mellitus. Am. J. Physiol. 243:E15-30.

12. DeFronzo, R. A., and E. Ferrannini. 1982. The pathogenesis of non-insulin-dependent diabetes: an update. Medicine. (Baltimore). 61:125-140.

13. Grunberger, G., S.-I. Taylor, R. F. Dons, and P. Gorden. 1983. Insulin receptors in normal and disease states. Clin. Endocrinol. Metab. 12:191-219.

14. Flier, J. S. 1983. Insulin receptors and insulin resistance. Annu. Rev. Med. 34:145-160.

15. DeFronzo, R. A. 1981. The effect of insulin on renal sodium metabolism: a review with clinical implications. Diabetologia. 21:165171. 966.

16. Editorial. 1982. Cells, ions, and blood-pressure. Lancet. II:965-

17. Clegg, G., D. B. Morgan, and C. Davidson. 1982. The heterogeneity of essential hypertension: relation between lithium efflux and sodium content of erythrocytes and a family history of hypertension. Lancet. II:891-894.

18. Cole, C. H. 1983. Erythrocyte membrane sodium transport in patients with treated and untreated essential hypertension. Circulation. 68:17-22.

19. De Luise, M., G. L. Blackburn, and J. S. Flier. 1980. Reduced activity of the red-cell sodium-potassium pump in human obesity. New Engl. J. Med. 303:1017-1022.

20. Klimes, I., M. Nagulesparan, R. H. Unger, S. L. Aronoff, and D. M. Mott. 1982. Reduced $\mathrm{Na}^{+}, \mathrm{K}^{+}$-ATPase activity in intact red cells and isolated membranes from obese man. J. Clin. Endocrin. Metab. 54:721-724.
21. Trevisan, M., D. Ostrow, R. Cooper, K. Liu, S. Sparks, A. Okonek, E. Stevens, J. Marquardt, and J. Stamler. 1983. Abnormal red blood cell ion transport and hypertension: the people's gas company study. Hypertension (Dallas). 5:363-367.

22. Moore, R. D. 1983. Effects of insulin upon ion transport. Biochim. Biophys. Acta. 737(1):1-49.

23. Cox, M., R. H. Sterns, and I. Singer. 1978. The defense against hyperkalemia: the roles of insulin and aldosterone. New Engl. J. Med. 299:525-532.

24. Friedman, S. M. 1983. Monovalent and divalent ions in vascular tissue. Ann. Intern. Med. 98:753-758.

25. Resh, M. D. 1982. Development of insulin responsiveness of the glucose transporter and the $\left(\mathrm{Na}^{+} \mathrm{K}^{+}\right)$-adenosine triphosphatase during in vitro adipocyte differentiation. J. Biol. Chem. 257:69786986.

26. Modan, M., H. Halkin, A. Karasik, and A. Lusky. 1984. Effectiveness of glycosylated hemoglobin, fasting plasma glucose and a single post load plasma glucose level in screening for glucose intolerance. Am. J. Epidemiol. 119:431-444.

27. DeFronzo, R. A. 1982. Glucose intolerance and aging. Diabetes Care. 4:493-501.

28. Fink, R. I., O. G. Kolterman, J. Griffin, and J. M. Olefsky. 1983. Mechanisms of insulin resistance in aging. J. Clin. Invest. 71:1523-1535.

29. National Diabetes Data Group. 1979. Classification and diagnosis of diabetes mellitus and other categories of glucose intolerance. Diabetes. 28:1039-1057.

30. Murphy, M. B., E. Kohner, P. J. Lewis, B. Schumer, and C. T. Dollery. 1982. Glucose intolerance in hypertensive patients treated with diuretics: a fourteen-year follow-up. Lancet. II:1293-1295.

31. World Health Organization. 1982. Summary report: WHO workshop on the methodology of hypertension cationic markers determination. Hypertension (Dallas). 4:916-917.

32. Cerasi, E., S. Efendic, and R. Luft. 1973. Dose-response relation between plasma-insulin and blood-glucose levels during oral glucose loads in prediabetic and diabetic subjects. Lancet. I:794-797.

33. Miettinen, O. S. 1976. Estimability and estimation in case referent studies. Am. J. Epidemiol. 103:226-235.

34. Flamenbaum, W. 1983. Metabolic consequences of antihypertensive therapy. Ann. Intern. Med. 98:875-880.

35. Pell, S., and A. D'Alonzo. 1967. Some aspects of hypertension in diabetes mellitus. JAMA. 202:10-16.

36. International Collaborative Group. 1979. Asymptomatic hyperglycemia and coronary heart disease: A series of papers by the international collaborative group, based on studies in fifteen populations. J. Chronic Dis. 32:683-837.

37. Wilson, P. W., D. L. McGee, and W. B. Kannel. 1981. Obesity, very low density lipoproteins, and glucose intolerance over fourteen years. Am. J. Epidemiol. 114:697-704.

38. Welborn, T. A., A. Breckenridge, A. H. Rubinstein, C. T. Dollery, and T. Russell Fraser. 1966. Serum-insulin in essential hypertension and in peripheral vascular disease. Lancet. I:1336-1337.

39. Berglund, G., and O. Andersson. 1981. Body composition, metabolic and hormonal characteristics in unselected male hypertensives. Intl. J. Obesity. 5(Suppl 1):143-150.

40. Voors, A. W., B. Radhakrishnamurthy, S. R. Srinivasan, L. S. Webber, and G. S. Berenson. 1981. Plasma glucose level related to blood pressure in 272 children, ages 7-15 years, sampled from a total biracial population. Am. J. Epidemiol. 113:347-356.

41. Florey, C. du V., S. Uppal, and C. Clowy. 1976. Relation between blood pressure, weight, and plasma sugar and serum insulin levels in school children aged 9-12 years in Westland, Holland. Br. Med. J. 1:1368-1371.

42. Zierler, K. L., and D. Rabinowitz. 1964. Effect of very small concentrations of insulin on forearm metabolism. Persistence of its action on potassium and free fatty acids without its effect on glucose. J. Clin. Invest. 43:950-962.

43. Craig, A. B., Jr., and P. L. Mendell. 1959. Blockade of 
hyperkalemia and hyperglycemia induced by epinephrine in frog liver and in cats. Am. J. Physiol. 197:52-54.

44. DeFronzo, R. A., P. Felig, E. Ferrannini, and J. Wahren. 1980. Effect of graded doses of insulin on splanchnic and peripheral potassium metabolism in man. Am. J. Physiol. 238:E421-427.

45. DeFronzo, R. A., R. S. Sherwin, M. Dillingham, R. Hendler, W. V. Tamborland, and P. Felig. 1978. Influence of basal insulin and glucagon secretion on potassium and sodium metabolism. Studies with somatostatin in normal dogs and in normal and diabetic human beings. J. Clin. Invest. 61:472-479.

46. McNair, P., S. Madsbad, C. Christiansen, M. S. Christensen, and I. Transbøl. 1982. Hyponatremia and hyperkalemia in relation to hyperglycemia in insulin-treated diabetic out-patients. Clin. Chim. Acta. 120:243-250.

47. Porter, G. A. 1983. Chronology of the sodium hypothesis and hypertension. Ann. Intern. Med. 98:720-723.

48. Tobian, L. 1983. Human essential hypertension: implications of animal studies. Ann. Intern. Med. 98:729-734.

49. MacGregor, G. A. 1983. Dietary sodium and potassium intake and blood pressure. Lancet. I:750-753.

50. Laragh, J. H., and M. S. Pecker. 1983. Dietary sodium and essential hypertension: some myths, hopes, and truths. Ann. Intern. Med. 98:735-743.

51. Stanton, J. L., L. E. Braitman, A. M. Riley, Jr., C-S. Khoo, and J. L. Smith. 1982. Demographic, dietary, life style, and anthropometric correlates of blood pressure. Hypertension (Dallas). 4(Suppl III):135-142.

52. Winquist, R. J., R. C. Webb, and D. F. Bohr. 1982. Vascular smooth muscle in hypertension. Fed. Proc. 41:2387-2393.

53. Christensen, N. J. 1983. Acute effects of insulin on cardiovascular function and noradrenaline uptake and release. Diabetologia. 25:377381 .

54. Epstein, F. H., and R. M. Rosa. 1983. Adrenergic control of serum potassium. New Engl. J. Med. 309:1450-1451.

55. Jenkins, D. J. A., R. H. Taylor, and T. M. S. Wolever. 1982. The diabetic diet, dietary carbohydrate and differences in digestibility. Diabetologia. 23:477-484.

56. Halverson, J. D., J. Kramer, A. Cave, A. Permutt, and J. Santiago. 1982. Altered glucose tolerance, insulin response and insulin sensitivity after massive weight reduction subsequent to gastric bypass. Surgery (St. Louis). 92:235-240.

57. Lohman, D., F. Liebold, W. Heilman, H. Senger, and A. Pohl. 1978. Diminished insulin response in highly trained athletes. Metab. Clin. Exp. 27:521-524.

58. LeBlanc, J., A. Nadeau, M. Boulay, and S. Rousseau-Migneron. 1979. Effects of physical training and adiposity on glucose metabolism and ${ }^{125}$ I insulin binding. J. Appl. Physiol. 46:235-239.

59. Vahouny, G. V. 1982. Conclusions and recommendations of the symposium on "Dietary Fibers in Health and Disease." Am. J. Clin. Nutr. 35:152-156.

60. Reisin, E., R. Abel, M. Modan, D. S. Silverberg, H. E. Eliahou, and B. Modan. 1978. Effect of weight loss without salt restriction on the reduction of blood pressure in overweight hypertensive patients. New Engl. J. Med. 298:1-6.
61. Hovell, M. F. 1982. The experimental evidence for weight-loss treatment of essential hypertension: a critical review. Am. J. Public Health. 72:359-368.

62. Paffenbarger, R. S., Jr., A. L. Wing, R. T. Hyde, and D. L. Jung. 1983. Physical activity and incidence of hypertension in college alumni. Am. J. Epidemiol. 117:245-257.

63. Bjorntorp, P. 1982. Hypertension and exercise. Hypertension (Dallas). 4(Suppl III):56-59.

64. Puska, P., A. Nissinen, E. Vartianinen, R. Dougherty, M. Mutanen, J. M. Iacono, H. J. Korhonen, P. Pietinen, U. Leino, S. Moisio, and J. Huttunen. 1983. Controlled, randomised trial of the effect of dietary fat on blood pressure. Lancet. I:1-5.

65. Fehily, A. M., J. E. Milbank, J. W. G. Yarnell, T. M. Hayes, A. J. Kubiki, and R. D. Eastham. 1982. Dietary determinants of lipoproteins, total cholesterol, viscosity, fibrinogen, and blood pressure. Am. J. Clin. Nutr. 36:890-896.

66. Glass, A. R., R. Bongiovanni, C. E. Smith, and T. M. Boehm. 1981. Normal valine disposal in obese subjects with impaired glucose disposal: evidence for selective insulin resistance. Metab. Clin. Exp. 30:578-582.

67. Fuller, J. H., M. J. Shipley, G. Rose, R. J. Jarrett, and H. Keen. 1980. Coronary-heart-disease risk and impaired glucose tolerance. The Whitehall Study. Lancet. I:1373-1376.

68. Kannel, W. B., and P. Sorlie. 1975. Hypertension in Framingham. In Epidemiology and Control of Hypertension. O. Paul, editor. Grune \& Stratton, Inc., Orlando, FL. 553-590.

69. Pyorala, K. 1979. Relationship of glucose tolerance and plasma insulin to the incidence of coronary heart disease: results from two population studies in Finland. Diabetes Care. 2:131-141.

70. Ducimentiere, P., E. Eschwege, L. Papoz, J. L. Richard, J. R. Claude, and G. Rosselin. 1980. Relationship of plasma insulin level to the incidence of myocardial infarction and coronary heart disease. Diabetologia. 19:205-210.

71. Welborn, T. A., and K. Wearne. 1979. Coronary heart disease incidence and cardiovascular mortality in Busselton with reference to glucose and insulin concentrations. Diabetes Care. 2:154-160.

72. Stamler, J. 1978. Lifestyles, major risk factors, proof and public policy. Circulation. 58:3-19.

73. Ashley, F. W., Jr., and W. B. Kannel. 1974. Relation of weight changes to changes in atherogenic traits: the Framingham Study. $J$. Chronic Dis. 27:103-114.

74. Editorial. 1983. Diet and ischemic heart disease-agreement or not? Lancet. II:317-318.

75. Morris, J. N., J. W. Marr, and D. G. Clayton. 1977. Diet and heart: a postscript. Br. Med. J. 2:1307-1314.

76. Burr, M. L., and P. M. Sweetnam. 1982. Vegetarianism, dietary fiber, and mortality. Am. J. Clin. Nutr. 36:873-877.

77. Paffenbarger, R. S., Jr., A. L. Wing, and R. T. Hyde. 1978. Physical activity as an index of heart attack risk in college alumni. Am. J. Epidemiol. 108:161-175.

78. Morris, J. N., M. G. Everitt, R. Pollard, S. P. Chave, and A. M. Semmence. 1980. Vigorous exercise in leisure time: protection against coronary heart disease. Lancet. II:1207-1210.

79. Stout, R. W. 1982. Is insulin atherogenic? Mt. Sinai J. Med. 49:223-226. 